\title{
Psychological and Physiological Stress in Hens With Bone Damage
}

\author{
Neža Rokavec and Manja Zupan Šemrov* \\ Department of Animal Science, Biotechnical Faculty, University of Ljubljana, Domžale, Slovenia
}

\section{OPEN ACCESS}

Edited by:

Michael Toscano,

University of Bern, Switzerland

Reviewed by:

Sabine G. Gebhardt-Henrich,

University of Bern, Switzerland

Christina Barbara Rufener,

University of California, Davis,

United States

Rebecca E. Nordquist

Utrecht University, Netherlands

${ }^{*}$ Correspondence:

Manja Zupan Šemrov

manja.zupansemrov@bf.uni-lj.si

Specialty section:

This article was submitted to Animal Behavior and Welfare,

a section of the journal

Frontiers in Veterinary Science

Received: 30 July 2020 Accepted: 12 November 2020 Published: 15 December 2020

Citation:

Rokavec N and Zupan Šemrov M

(2020) Psychological and

Physiological Stress in Hens With

Bone Damage.

Front. Vet. Sci. 7:589274

doi: 10.3389/fvets.2020.589274
Abnormalities in bone development in humans and non-humans can lead to impaired physical and psychological health; however, evidence is lacking regarding the role of individual psychosocial factors in the development of poor bone conditions. Addressing this lack of knowledge, we used low-productive laying hens $(n=93)$ and assessed behavioral responses to an open-field test [at 17, 18, 29, 33 weeks of age (wa)], an aerial predator test (at $39 \mathrm{wa}$ ), and a social reinstatement test (at $42 \mathrm{wa}$ ). Bone condition was assessed using a palpation technique on five occasions (at 16, 29, 33, 45, 58 wa), with half of the hens experiencing damage (deviations, fractures, or both) at 29 wa and all hens by 58 wa. Corticosterone (CORT) concentration in feathers (at 16, 33, 58 wa) and body weight (at 23, 47, $58 \mathrm{wa}$ ) were also investigated. We hypothesized that lighter birds (at 23 wa) with higher CORT (at 16 wa) and open field-induced fear collected before the onset of lay (at 17 and 18 wa) are associated with a worse bone condition when in lay. We also hypothesized that those birds with more damage at the peak of laying (at 29 wa) would be lighter at 47 and 58 wa and more fearful by showing higher open fieldinduced (at 29 and 33 wa) and predator-induced fear responses, however, acting less socially toward conspecifics. These hens were also expected to have higher CORT (at 33 and 58 wa). Our results show no association between open-field fear level and fear behavior, CORT concentration, or body weight on the one hand (all measured before starting to lay) and bone damage at 29 wa on the other. When in lay, bone damage was associated with more pecking and less crossing zones when faced with an open-field situation at 29 wa and improved sociality at 42 wa. This study provides the first evidence of a relationship of bone health with fear, sociality, and stress response. When in poor bone condition, our hens had enhanced psychological stress measured by fear behavior reactivity but not physiological stress measured as feather CORT concentration.

Keywords: keel bone, poultry, stress physiology, behavior, body development, affective state

\section{INTRODUCTION}

Bone disease, such as osteoporosis in humans, is often seen as a silent disorder until it causes fractures (1). Yet, the consequence of such disease can have a major impact on individuals such as a decrease in physical and psychological health. Many humans who suffer from bone fractures experience significant pain and weight loss; they may lose the ability to stand and walk (2) or may be immobilized by a fear of falling (3) or even begin to feel isolated and helpless (2). On top of these effects, an increase in indirect costs [e.g., lost productivity for patients and caregivers (2) and increased stress level (4)] has recognized. 
Animal welfare scientists agree that laying hens suffer from a variety of welfare problems, including the keel bone damage (KBD) (5), which is estimated to reach a prevalence of between 30 and $90 \%$ by 45 weeks of age (wa) when the ossification of the keel bone (KB) is completed (6-8). KBD includes both fractures and deviations (9). Unlike fractures, which usually happen during an isolated event such as crashes/collisions during flight or uncontrolled landings and takeoffs (9), the development of deviations happens over a period of time as an outcome of bone remodeling in response to regular loading pressure during roosting (7). Bone damage is known to affect a broad spectrum of issues in the poultry industry, including egg production $(8,10,11)$, water intake (12), body weight (6), deformation of breast muscle (12), and to cause welfare problems (9) including pain (13). In 1868, Darwin became the first to document (14) that egg-producing domestic fowl, laying fewer eggs than the hens nowadays, exhibited KBs that were moderately crooked or extremely deformed. In the early 1990s, crooked keels in laying hens were ascribed to hereditary disease (15), rickets (16), faulty metabolism, or a slow process of ossification (17). Despite this, only recently has research intensively focused on looking at the underlying causes and consequences of KBD in commercial laying hens.

Recent findings $(10,12,18)$ reveal that it is likely that production is just one of many factors affecting bone integrity, explaining why some studies found no effect on egg production $(6,12)$ or body weight (19). By reviewing several research studies, Riber et al. (9) summarized that psychological stress factors may be related to bone damage and that $\mathrm{KBD}$ promotes the expression of negative states. In layers, investigating negative affective states focused mainly on pain $(13,19)$ or the fear level (20-22), often related to fear of humans (22), while for positive states, it concentrated on assessing social behavior (21). Studies also found that bone damage affects not only welfare but physiological parameters (10), although the problem's multifactorial nature makes it difficult to study the underlying causes and consequences of KBD in commercial laying hens.

To date, insufficient longitudinal data have been available to link bone damage and emotional consequences or to investigate the hypothesis that the affective state may impact damage. Riber and Hinrichsen (23) suggested, albeit have not yet clearly demonstrated, that a link exists between injurious pecking damage, bone damage, and fearfulness. Recently, by investigating changes in the hippocampus in a small number of commercial Lohmann Brown hens (15 hens with severe and nine hens with minimal $\mathrm{KB}$ fractures) in an aviary system, Armstrong et al. (24) found that hens with KB fractures are more likely to experience negative affective states that last for at least 34 weeks. In line with this and the fact that fearfulness of an individual, which is a known measure of psychological stress and thus, a negative affective state, could affect physical health (25) and its sensitivity to physiological stress (26), our main objective was to investigate the relationships between the affective state recorded during behavioral testing and the development of bone condition, corticosterone (CORT) concentration, and body weight. Levels of CORT deposited in feathers were analyzed to provide a measure of longer term physiological stress [i.e.,
$(26,27)]$. Slovenian locally adapted laying hens of the Styrian breed $(\mathrm{n}=93)$ were subjected to standardized test situations [i.e., open-field test (OFT), aerial predator test (APT), social reinstatement test (SRT)], and the level of fear and sociality were measured. We chose this particular strain of bird to improve our understanding of the behavioral and stress responses of hens with low egg production and good resistance to diseases (28); their bone condition is expected to be less likely poor and to show greater variation in behavioral responses compared to highly productive hens that have been intensively selected. Moreover, thus far, there are no data on the association between the prevalence of KBD in non-commercial chicken breeds and affective states. Assuming lighter birds are more prone to show fear behavior and be fearful (29), fearful birds have a higher risk of injuries (30), and bone development depends on the concentration of glucocorticoids in humans (2) and animals (18, 31 ), we first hypothesized that lighter individuals that show more fear-related characteristics and a higher stress-induced CORT before starting to lay will have a poorer bone condition at a later time point and, second, that those birds with more damage at the peak of laying will show more fear but act less socially toward conspecifics and have a lower body weight and a higher CORT. The latest hypothesis was derived from human studies (24) but also from the suggestion that when small prey animals are subjected to fear stimuli such as predator-like stimulus, this may elevate long-term stress and defensive responses and may lead to future stress-induced weight loss (32).

\section{MATERIALS AND METHODS}

\section{Animals and Housing}

The experiment was conducted from October 2017 to August 2018 at the Krumperk Educational and Research Centre, University of Ljubljana, Biotechnical Faculty. Randomly selected pullets $(n=93)$ and cockerels $(n=15)$ were obtained from a commercial flock of a basic floor-rearing system at 16 wa and transported to the laying pen $(1 \times \mathrm{w}=865 \times 496 \mathrm{~cm})$. From 16 to 58 wa, the flock was kept in this barn system with wood shavings (7-cm depth) and started to lay at 23 wa. To allow recognition, all females were marked with leg rings. The laying pen was divided by a wire mesh into a smaller $(1 \times \mathrm{w}=865 \times$ $186 \mathrm{~cm})$ and a larger $(1 \times \mathrm{w}=865 \times 310 \mathrm{~cm})$ area linked by an always-open door. Light was provided by two bulbs according to a 14:10 h light:dark cycle. Chickens had free access to a standard commercial layer diet from three round feeders (at $27 \mathrm{~cm}$ height) and water from drinking lines (at $37 \mathrm{~cm}$ height) with 25 water nipples in the smaller area and 29 in the larger area. Three wooden perches were placed in the middle of the larger area, each with dimensions of $190 \times 4 \times 6 \mathrm{~cm}$, placed at a height of $66 \mathrm{~cm}$ above the ground. The pen contained two metal nest box lines at a height of $50 \mathrm{~cm}$ above the ground when measured from the lower line, with 14 nest boxes each $(\mathrm{w} \times \mathrm{d} \times \mathrm{h}=30$ $\times 30 \times 30 \mathrm{~cm})$ and three wooden perches $(1 \times \mathrm{w} \times \mathrm{h}=200$ $\times 4 \times 2 \mathrm{~cm})$ in front. The available perch space was $12.9 \mathrm{~cm}$ per bird. Two automatic axial propeller fans were used to draw air out of the building through the wall vents (negative pressure 


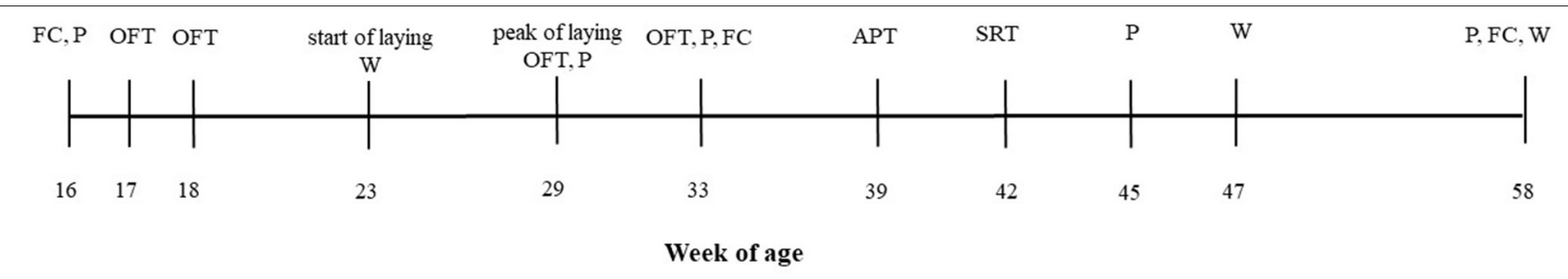

FIGURE 1 | The timeline of the measurements taken. FC, feather collection; P, palpation; W, body weight; OFT, open-field test; APT, aerial predator test; SRT, social reinstatement test.

ventilation), and two air inlets were used to ensure fresh air entered the barn.

\section{Experimental Design}

Figure 1 illustrates the different experimental procedures carried out over a period of 42 weeks, during which the hens were individually weighed (at 23, 47, $58 \mathrm{wa}$ ), exposed to three different behavioral tests, and palpated to record $\mathrm{KB}$ status. To obtain a retrospective measure of the long-term stress experienced by the birds during feather growth $(27,33)$, feathers were taken at three time points for analysis of CORT concentrations. These procedures are explained in more detail in the following sections and were chosen to investigate the relationships between fear as an indicator of psychological stress, CORT as an indicator of physiological stress, and KBD as well as body weight as a physical condition.

\section{Palpation of the Keel Bone}

The presence of both fractures and deviations of the $\mathrm{KB}$ was assessed by palpation on five occasions (at 16, 29, 33, 45, and 58 wa) using the Simplified Keel Assessment Protocol (SKAP) palpation system (34). At 16 and 29 wa, only the presence or absence of damage was recorded, whereas at 33, 45, and 58 wa, the type of damage (deviation, fracture, or both) was also specified. The person assessing the damage was trained on how to palpate hens during a 2-day course at the University of Bern in 2017. The study of this training school revealed that training with radiographs improved palpation accuracy (35). On each assessment occasion, hens were taken from the pen in random order and transferred to a nearby room. Each hen was held in the observer's arms in the position of a cradle and the ventral and lateral surfaces of the $\mathrm{KB}$ were palpated by running the forefinger and thumb up and down the bone.

\section{Feather Collection and Corticosterone Analyses}

Feather collection was done by cutting the primary third feather of the wing from each hen. The first feather was representative of the period before the onset of lay and cut out at 16 wa. The second feather was cut at 33 wa, i.e., 4 weeks after the peak of lay, and the final one at the end of the experiment ( $58 \mathrm{wa}$ ). The first and the third feathers (that grew out between 16 and 58 wa) were taken from the left wing and the second feather from the right wing. Feathers were stored separately in a paper envelope and kept on a shelf at ambient indoor temperature before analysis. A methanol-based extraction technique was used to extract CORT from feathers [adjusted after Bortolotti et al. (27)]. The feathers were prepared by cutting vanes into pieces with scissors. From each feather, $30 \mathrm{mg}$ was used and put into a test tube. Then, $10 \mathrm{ml}$ of methanol gradient grade for liquid chromatography (Merck KGaA, Darmstadt, Germany) was added, and the samples were placed in a sonicating water bath at room temperature for $30 \mathrm{~min}$, followed by incubation at $50^{\circ} \mathrm{C}$ overnight in a shaking water bath. The methanol was then separated from the feather material by basic gravity filtration using a cellulose filter paper in the filtration funnel. The feather remnants, original sample vial, and filtration material were washed twice with $\sim 2.5 \mathrm{ml}$ of additional methanol; the washes were added to the original methanol extract. The methanol extract was placed in a $50^{\circ} \mathrm{C}$ water bath and subsequently evaporated in a fume hood under nitrogen gas. Evaporation of the samples was completed within a few hours, and the extract residues were reconstituted in $500 \mu \mathrm{l}$ of $15 \%$ methanol. Reconstituted samples were frozen at $-20^{\circ} \mathrm{C}$ until analyzed for CORT. The concentration of the samples (nmol/L extract) was assayed using the commercially available ELISA kit (DE4164, Kiel, Germany). The test procedure followed standard methods. While calculating the CORT concentration in feathers ( $\mathrm{pM} / \mathrm{g})$, the dilution factor (16.67) was taken into account. The CORT concentration represented three measures (CORT at 16 wa represented the storage between 0 and 16 wa; CORT at 33 wa represented the storage between 0 and 33 wa; CORT at 58 wa was the sum of CORT at 16 wa and CORT at 33 wa).

\section{Behavioral Tests}

Starting at $17 \mathrm{wa}$, the hens were subjected to several behavioral tests. The tests were performed in the same order for each hen, i.e., first the OFTs, then APT, and finally the SRT. The OFT was performed four times at different ages in order to investigate intra-situation coping responses. As for inter-situation behavioral responses that may represent generalized fearfulness, APT and SRT were performed at later ages but were not tested in weeks in which palpation or feather collection were carried out in order to avoid confounding the behavioral readouts. Consistency of behaviors in different tests was investigated. Hens were individually caught from the pen, each time from a different location in order to avoid biases in the test order as less fearful or slower birds are often picked 


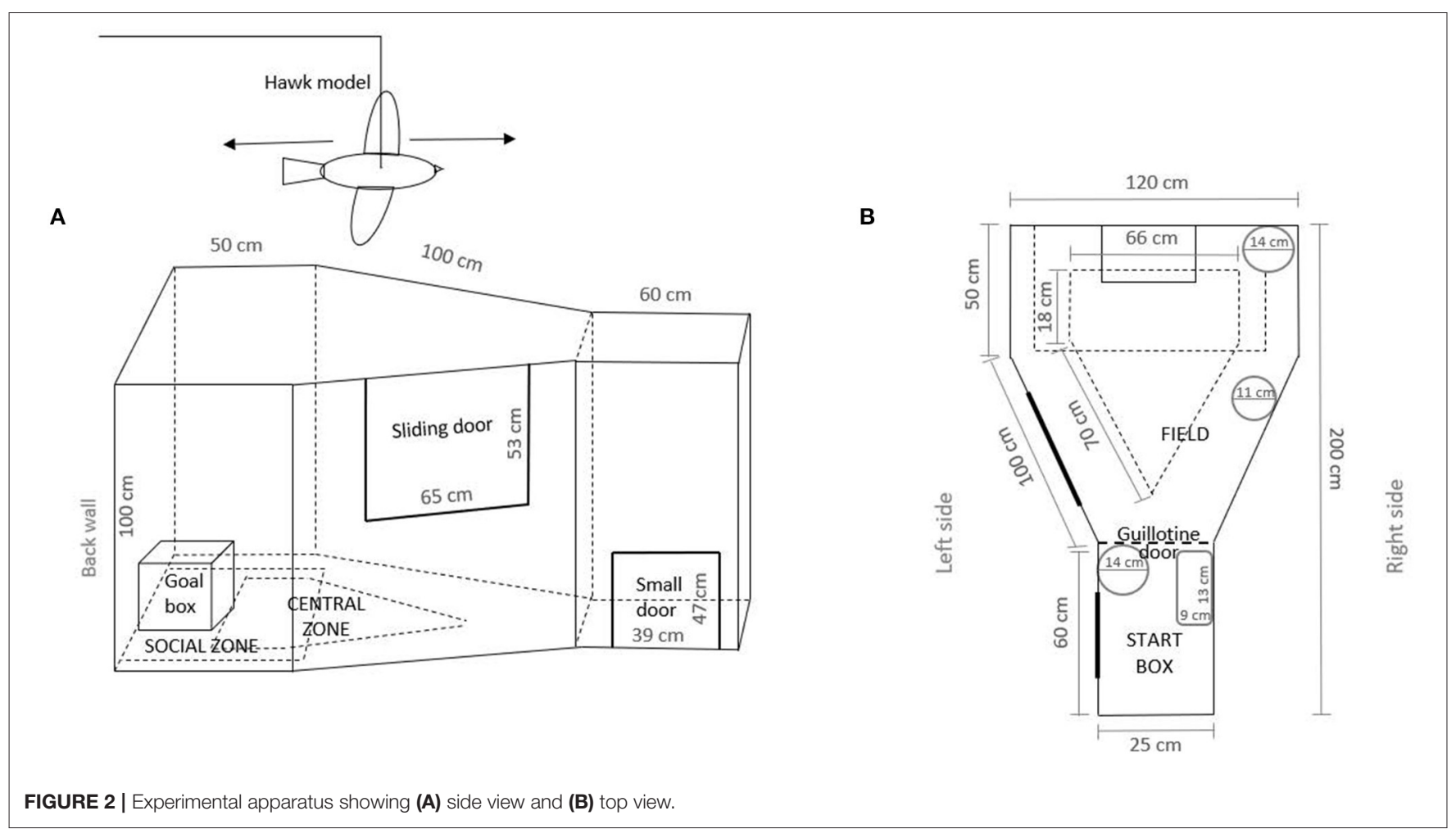

first, and brought to the test apparatus in one person's hands. Their behaviors were recorded using direct observations by one observer. Recorded by stopwatch, timing started $30 \mathrm{~s}$ after being placed in the test apparatus.

\section{Test Apparatus}

The apparatus (Figure 2) was a weather-resistant black plywood (T-fix) trapezoid-like arena isolated from humans and animals [adjusted after de Haas et al. (36)]. It was located in a 474 $(\mathrm{l}) \times 360(\mathrm{w}) \times 258 \mathrm{~cm}(\mathrm{~h})$ room next to the laying pen and illuminated with a light bulb of $206.9 \mathrm{~lx}$ (measured at the hen's head). An extra light was placed $140 \mathrm{~cm}$ above the apparatus to help track the test hen and ensure visibility. A hen was placed in the start box through a small door and then introduced into the field arena when the guillotine door of the start box was opened. Observations started when the guillotine door was opened. To measure movement, the arena was divided into zones (i.e., central and social zone) marked with black tape. A human could gain access through the sliding door to catch a bird and to clean (i.e., vacuuming feathers and wood shavings and absorbing feces with cellulose) the area after each testing. By using mirrors, the observer standing behind the start box was out of view of the test subjects so as not to influence their behavior. The circular and rectangular mirrors were placed above the guillotine door of the start box. Other circular mirrors were on the back wall and on the right side of the apparatus. Part of the scoring criteria included flying out of the arena, which is why the top of the arena was not covered.

\section{Open-Field Test}

The hens $(n=93)$ were individually exposed to an OFT at $17,18,29$, and 33 wa between 9:00 and 15:00 h. Each of them lasted $3 \mathrm{~min}$. At 10, 20,30,40,50, and $60 \mathrm{~s}$ after opening the guillotine door, the birds' fear responses were categorized as calm, ambiguous, fearful, and highly fearful according to specific behaviors detailed in predetermined selection criteria (Table 1), and the fear scores were averaged across the six observations.

Other fear behaviors (Table 2) were recorded for $3 \mathrm{~min}$ of testing using continuous sampling. Pecking and preening were added because they frequently occurred during the pilot study.

TABLE 1 | Selection criteria for fear responses in the open field test.

\begin{tabular}{llc}
\hline $\begin{array}{l}\text { Fear } \\
\text { response }\end{array}$ & Behavior & $\begin{array}{c}\text { Fear } \\
\text { score }\end{array}$ \\
\hline Calm animal & $\begin{array}{l}\text { Exploring, standing or walking, short or } \\
\text { normal length of the neck, and no } \\
\text { vocalizing or vocalizing quietly (calm, low) }\end{array}$ & 20 \\
Ambiguous & $\begin{array}{l}\text { Standing or walking, neck stretched, head } \\
\text { flicks, and no vocalizing or vocalizing }\end{array}$ & 40 \\
Fearful animal & $\begin{array}{l}\text { quietly (calm, low) } \\
\text { Standing or walking, neck stretched, head } \\
\text { flicks, and vocalizing loudly } \\
\text { Highly fearful }\end{array}$ & $\begin{array}{l}\text { Escape, attempting to escape, and } \\
\text { vocalizing loudly or no vocalizing. The bird } \\
\text { is completely still (freeze behavior). }\end{array}$ \\
\hline
\end{tabular}

Fear scores were adapted from Agnvall et al. (37). Fear responses and behaviors were newly defined. 


\section{Aerial Predator Test}

Behavior was observed during a simulated aerial predator attack that was carried out once at $39 \mathrm{wa}$, between 9:00 and 14:00 h, to investigate the initial response to a potential natural predator. For behaviors (Table 2), we used instantaneous sampling with 10 -s intervals during 2 min of testing, while continuous sampling was used for latency to escape. In order to obtain a baseline of the behavior, the animals were first observed undisturbed. After $1 \mathrm{~min}$ of opening the guillotine door, a hawk-silhouette model (Figure 2) (measuring $41.0 \times 22.4 \mathrm{~cm}$ ) made out of browncolored plywood (natural color of the falcon) was pulled back and forth along a string starting $140 \mathrm{~cm}$ above the testing room floor, $15 \mathrm{~cm}$ from the back wall of the apparatus. The model passed through the arena's $340 \mathrm{~cm}$ in $3 \mathrm{~s}$. Before and after the simulated overflight, the hawk silhouette was hidden behind a gray curtain.

\section{Social Reinstatement Test}

At 42 wa, the hens' level of sociality (motivation to be with conspecifics) was measured between 8:00 and 12:00 h. Beside the back wall of the arena, one stimulus hen familiar to the test bird (one of 93 test hens) and of the same age, was kept in a wooden framed box (Figure 2) of $30(\mathrm{l}) \times 40(\mathrm{~d}) \times 40(\mathrm{~h})$ made of wire mesh. The stimulus bird was changed after each test. An area close to the social companion was defined as a social zone, marked with black tape at $25 \mathrm{~cm}$ around the goal box. This distance was chosen according to Dawkins (38), who claims that social recognition in hens may only occur at distances $<30 \mathrm{~cm}$. The hens were tested once. The test procedure was as follows: when the test started, the behaviors described in Table 2 were observed for 3 min using continuous sampling.

\section{Statistical Analysis}

Five hens unexpectedly and unrelated to the experiment died before 58 wa and were excluded from certain analyses. Further, feather CORT concentration was missing for four hens at 16 wa and for one hen at 33 wa. The statistical analysis was performed using the SAS/STAT software, version 9.4, of the SAS System for Windows (C) 2002-2012 SAS Institute Inc. The normal distribution for quantitative traits was determined by the Shapiro-Wilk test. All reported $P \leq 0.05$ were classified as statistically significant while $<0.06$ as with a strong tendency.

Considering the behavioral observations, latencies to leave the start box and to reach the central zone among repetitions of OFT were analyzed in a survival analysis context (39) by the PHREG procedure, where non-events were treated as right-censored data. Preening and pecking as well as APT behaviors were calculated first as frequencies per hen and then as number of times per time period for all the tested birds. A difference between the behaviors observed in OFTs, as well as before and after a hawk appearance in APT, was investigated using the chi-square test. Consistency was computed for behaviors in repeated OFTs as suggested by

TABLE 2 | Ethogram of the behaviors recorded during the tests and their descriptions.

\begin{tabular}{|c|c|c|}
\hline Test & Behavior & Description \\
\hline \multicolumn{3}{|l|}{ OFT } \\
\hline & Latency to leave the start box ${ }^{D}$ & Length of time from the start of testing to stepping in the field with both feet \\
\hline & Latency to reach the central zone $^{D}$ & Length of time when both feet reach into the central zone \\
\hline & Crossing the central zone ${ }^{F}$ & Defines how often the hen crosses the central zone \\
\hline & Preening ${ }^{F}$ & Defines how often the hen moves its head in a smoothing motion over the body \\
\hline & Pecking $F$ & Defines how often the hen pecks on the ground or at the wall of the platform as visual inspection \\
\hline \multicolumn{3}{|l|}{ APT } \\
\hline & Activity ${ }^{F}$ & $\begin{array}{l}\text { Defines how often the hen has a relaxed body stance, short or normal length of the neck (when she moves, } \\
\text { stands, or sits) and does not vocalize or vocalizes quietly }\end{array}$ \\
\hline & Freeze ${ }^{F}$ & Defines how often the hen is completely still ${ }^{\star}$ \\
\hline & Escape attempt ${ }^{F}$ & $\begin{array}{l}\text { Defines how often the hen tries to escape, i.e., constantly looks up at the top of the platform with neck } \\
\text { stretched or tries to fly out }\end{array}$ \\
\hline & Being alert ${ }^{\mathrm{F}}$ & $\begin{array}{l}\text { Defines how often the hen has an alert body stance with neck stretched (when she moves, stands, or sits) } \\
\text { and does not vocalize or vocalizes loudly }\end{array}$ \\
\hline & Latency to escape ${ }^{\mathrm{D}}$ & Length of time from the start of testing to the platform breakout \\
\hline \multicolumn{3}{|l|}{ SRT } \\
\hline & Vocalization ${ }^{F}$ & Defines how often the hen vocalizes \\
\hline & Latency to vocalize ${ }^{\mathrm{D}}$ & Length of time to the first sound the hen makes \\
\hline & Latency to escape ${ }^{\mathrm{D}}$ & Length of time from the start of testing to the platform breakout \\
\hline & Latency to leave the start box ${ }^{D}$ & Length of time from the start of testing to stepping in the field with both feet \\
\hline & Latency to reach the social zone ${ }^{\mathrm{D}}$ & $\begin{array}{l}\text { Length of time when at least one of the feet reaches the social zone or a hen jumps from the field on the cage } \\
\text { located in the social zone }\end{array}$ \\
\hline & Duration in social zone ${ }^{\mathrm{D}}$ & Time spent in the social zone* \\
\hline
\end{tabular}

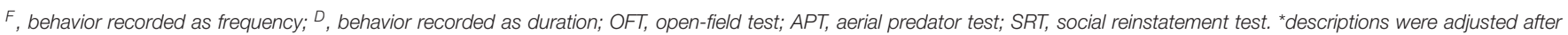
Agnvall et al. (37). 
Nakagawa and Schielzeth (40), where behaviors were treated as binary variables with 1 (i.e., event) and 0 (i.e., non-event). A multinomial overdispersion model was used in the GLIMMIX procedure, and consistencies are presented on a latent scale.

In the following step, the KBD as a binary outcome (1, damage; 0 , no damage) and CORT concentration as a continuous variable at different ages were analyzed by the FREQ procedure using the $\mathrm{CMH}$ option in the TABLES statement or by the MIXED procedure with a hen treated as a random effect, respectively. Since the majority of hens experienced KBD at 33 wa $(80.6 \%)$ and all hens at 58 wa $(100.0 \%)$, it was only possible to use KBD data at the peak of lay (29 wa), where $50 \%$ of hens were found with KBD for further analysis. Using the LOGISTIC procedure with modeling probability that KBD is 1 , we investigated if the average fear score at 17 (OFT1) and 18 wa (OFT2) and fear-related behavior responses in OFT1 and OFT2, body weight at 23 wa, and CORT at 16 wa affected KBD. We were further interested to know if the presence/absence of KBD was related to the average fear score at 29 (OFT3) and 33 wa (OFT4), body weight at 47 and 58 wa, CORT at 33 and 58 wa, and the behavior responses displayed in OFT, APT, and SRT. In the original models, all of the tested variables were included, but those found not significant for KBD were removed. Wald chi square statistics was provided for results deriving from the LOGISTIC procedure.

\section{RESULTS}

\section{Behavioral Tests Open Field Test}

With repetitive exposure to the OFT, more hens left the start box $\left(\chi^{2}=25.43, \mathrm{df}=4, P<0.0001\right)$ and did so faster $\left(\chi^{2}=72.02, P\right.$ $<0.0001$; data not shown). The highest number of hens reached the central zone in OFT3 $\left(\chi^{2}=17.75, \mathrm{df}=3, P=0.0005\right)$, and they did so more frequently in OFT3 and OFT4 than in OFT1 and OFT2 $\left(\chi^{2}=40.62, \mathrm{df}=3, P<0.0001\right.$; data not shown $)$. There were six hens that did not leave the start box in any of the four repetitions. The frequencies of preening (from OFT1 to OFT4 test; $1: n=57,2: n=104,3: n=72,4: n=62 ; \chi^{2}=18.13$, $\mathrm{df}=3, P=0.0004$ ) and pecking (from OFT1 to OFT4 test; $1: n$ $=104,2: n=150,3: n=127,4: n=145 ; \chi^{2}=9.89, \mathrm{df}=3, P=$ 0.02 ) also differed among repetitions.

Consistency of behaviors treated as binary traits in repeated OFTs was low to moderate. Leaving the start box over four repetitions of OFT had consistency of 0.181 , and with inclusion of SRT, it increased to 0.188 . If only records from the rearing period were considered, consistency was 0.211 , and for records from only the laying period, it was 0.561 . Consistency for reaching the central zone was lower than consistency for leaving the start box, with higher values in the rearing and laying period separately compared to the inclusion of all four repetitions of OFT. Preening had the highest consistency of the behaviors observed; 0.345 for four repetitions of OFT, 0.442 in the rearing and 0.493 in the laying period. Consistency for pecking was 0.159 for four repetitions of OFT, 0.093 in the rearing and 0.289 in the laying period.

\section{Aerial Predator Test}

The hens' behavior differed when comparing responses before and after the appearance of the hawk. More exploring before its appearance ( 283 vs. 172 times; $\chi^{2}=27.08, \mathrm{df}=1, P<0.0001$ ) and less standing alert (139 vs. 202 times; $\chi^{2}=11.64, \mathrm{df}=1, P=$ $0.0006)$ were observed. No differences were observed in freezing behavior ( 89 times before vs. 111 times after; $\chi^{2}=2.42, \mathrm{df}=1$, $P=0.12$ ) and escape attempts ( 31 times before vs. 32 times after; $\chi^{2}=0.02$, df $=1, P=0.90$ ). Some hens froze (before: $n=22$; after: $n=27)$, and others tried to escape the test apparatus $(n=$ 18), but few managed to escape (before: $n=5$; after: $n=3$ ).

\section{Social Reinstatement Test}

In the SRT, hens $(n=78)$ left the start box in $23.85 \pm 3.30 \mathrm{~s}$, 53 hens reached the social zone in $63.21 \pm 6.12 \mathrm{~s}$ and stayed in the zone for $100.75 \pm 6.98 \mathrm{~s}$. They needed $55.42 \pm 7.07 \mathrm{~s}$ to start vocalizing ( $n=48$ hens), with the maximum number of events per hen being 18 . Some hens $(n=10)$ escaped from the test apparatus with a latency of $49 \pm 14.79 \mathrm{~s}$.

\section{Keel Bone Damage}

The number of hens exhibiting bone damage (deviations and fractures combined) increased with age (Mantel-Haenszel $\chi^{2}=$ 21.86, $\mathrm{df}=1, P<0.0001)$, with most hens without KBD at 16 wa $(6.5 \%)$, half of the hens with $\mathrm{KBD}$ at 29 wa (50.4\%), almost all hens at $45 \mathrm{wa}(94.6 \%)$, and all hens showing $\mathrm{KBD}$ at 58 wa (100\%). CORT concentration was also found to increase with age $($ mean $\pm S D ; 16$ wa $=44.78 \pm 16.07 ; 33$ wa $=67.83 \pm 29.73$; 58 wa $=96.95 \pm 25.03 ; F=696.81, \mathrm{df}=2, P<0.0001)$. Hens weighed $1435.05 \pm 164.11 \mathrm{~g}$ at $23 \mathrm{wa}, 1795.22 \pm 213.24 \mathrm{~g}$ at 47 wa, and $1825.39 \pm 226.33 \mathrm{~g}$ at 58 wa.

Before starting to lay and by using a logistic regression model, we found that body weight affected KBD by lighter hens showing more bone damage (Wald $\chi^{2}=4.65, P=0.03$ ), but biological significance was negligible (for a $1 \mathrm{~kg}$ heavier hen probability increased by only $0.3 \%$ ). CORT stored from 0 to 16 wa had no relationship to KBD at 29 wa (Wald $\chi^{2}=0.17, P=0.68$ ) nor had the average fear score at 17 (Wald $\chi^{2}=1.28, P=0.26$ ) or 18 wa (Wald $\chi^{2}=0.49, P=0.48$ ). When in lay, pecking and frequency of crossing zones at 29 wa in OFT3 as well as latency to leave starting arena in SRT showed a relationship with KBD (Table 3). Hens with bone damage at 29 wa were pecking more but crossing zones less in the OFT and reaching the testing arena faster in SRT. No other relationships were found including those between KBD and average fear scores (data not shown).

\section{DISCUSSION}

This study used fowl as an animal model to investigate the relationship between fear and stress responses and bone health. We found multiple relationships. Although we cannot confirm that the patterns we observed in the predator- and open fieldinduced fear situations are individual behavioral strategies stable over a longer time, our results support the existence of a relationship between psychological stress experienced as fear and the development of physical health reflected in bone condition. 
TABLE 3 | Behavior responses as frequency (mean \pm SE) associated with KBD at 29 week of age (wa) in OFT and SRT.

\begin{tabular}{|c|c|c|c|c|}
\hline & \multicolumn{4}{|c|}{ Chi-Square } \\
\hline Pecking ${ }^{O F T}$ & $1.64 \pm 0.33$ & $1.09 \pm 0.24$ & 3.78 & 0.052 \\
\hline Crossing the central zone OFT & $1.13 \pm 0.18$ & $1.41 \pm 0.21$ & 5.81 & 0.02 \\
\hline LLAS $(S)^{\text {SRT }}$ & $17.73 \pm 3.41$ & $31.76 \pm 5.94$ & 6.62 & 0.01 \\
\hline
\end{tabular}

$K B D$, keel bone damage; OFT, open-field test; SRT, social reinstatement test; LLAS, latency to leave the start box.

In contrast, we were unable to confirm that bone condition was associated with either physiological stress (measured as feather CORT concentration) or body weight.

In the OFT, the individual behavior was determined by different quantities of fear behavior (as number of times the target behavior was observed; Table 2) and was inconsistent with time, however, associated with bone condition at the peak of laying period (at 29 wa). This means that an individual used a strategy on an ad hoc basis based on how good/bad its keel health was. The behavior displayed seems to depend also on an individual life stage need (41) or may result from an increased willingness to move from the start box or from a habituation effect $(42,43)$, since being in lay led to an increase in the number of our hens leaving the start box and reaching the central zone. This change might also depend on age or early experience because fearful shyness occurs among the young of most mammalian species (44), which could also be true for the bird. Considering the impact of affective states on bone damage, our results show that fear responses categorized from calm to highly fearful, a high psychological state of fear at 17 and $18 \mathrm{wa}$, but also high physiological stress assessed by feather CORT concentration (at 33 and 58 wa) or low body weight (at 47 and 58 wa) were not associated with less bone damage at 29 wa, which is contrary to our expectations. As reviewed by Harlander-Matauschek et al. (30), it could be that with regard to $\mathrm{KB}$ fractures, fearful hens may be more likely to panic and thus collide with pen furnishing, leading them to develop worse bone health. Our current results are incongruent with expectations based on this literature. However, no study has been reported to clearly investigate and demonstrate the link between underlying fearfulness and bone damage in animals. Still, one possible reason for not confirming our first hypothesis is the exposure of our hens to only two acute fear-induced situations before the bone damage reached the prevalence of half of the hens. One could also argue that the responses recorded are unrepresentative of actual fearfulness because they were not investigated in the hen's home pen.

Considering the consequences of bone damage, hens with bone damage at the peak of the laying period ( $29 \mathrm{wa}$ ) had similar feather CORT concentrations at 33 and 58 wa and body weight at 47 and 58 wa. When exposed to open field-induced fear situation at $29 \mathrm{wa}$, these hens were recorded as having moved less often between zones in the test arena. According to the assumptions of the OFT (45), this can be a measure of worse locomotor behavior in chickens. Since these hens also showed more pecking on the ground and the wall, it is less likely that this behavior is a sign of exploratory pecking. Given the presence of escape attempts (recorded as fear responses and labeled as highly fearful animals), these responses suggest that they perceived the situation as more threatening or fearful. These findings also suggest that fearfulness, bone damage, and pecking behavior are related, a link proposed previously with injurious pecking behavior (23).

In many species, fear level has been negatively correlated with social motivation [birds (46), pigs (47), horses (48)], and this has been linked to high physiological stress responses [birds (49), pigs (50), humans (4)]; however, our results contradict these links. In the social situation at $42 \mathrm{wa}$, the responses of hens with bone damage suggest improved sociality with animals leaving the start box. According to models of motivation (51), these responses may indicate that individuals had a higher motivation to explore a subject/environment or a lower fear or anxiety level in a social context. However, this result may also be interpreted as a sign of fast decision-making (52) or boldness (53) with active seeking to escape and social reinstatement (54) or sensitization (55). When coupled with the argument of Mills et al. (46) that social motivation predominates over the fear response in individuals with a high tendency of making social contact, hens with bone damage imply to develop a different biological sensitivity to the social context (44) compared to hens without damage. Whatever the reason, it appears that bone damage, which potentially causes pain, particularly when bone is broken $(5,9)$, leads to different fear- and social-related psychological stress in birds, but not necessarily stress-induced CORT. It must be emphasized that this interpretation is based on an analysis where fractures and deviations were considered in a single variable due to the method applied, which is most practical from a commercial perspective but is not reliable enough to detect all differences in damage nor the time of a fracture. Evidence suggests that bone fractures have a negative impact on self-esteem, body image, and mood in humans (2) as well as negative affective states in laying hens (24).

It has also been documented that humans $(2,56)$ and animals [dogs (57), chicken (58), mice (59)] can encounter problems with bone condition due to body weight. It remains unclear why in species, such as birds, the KB is not under the influence of body weight, although it is in conjunction with the studies of Nasr et al. $(13,19)$ using a highly productive Lohmann Brown laying strain. One possible explanation for not detecting its influence could be the low variation in body weight found in this study.

It also remains unsettled why after the ossification is completed, at $45 \mathrm{wa}$, all hens ended up with a bone deviation or fracture, which seems to be a general phenomenon $(7,60)$. They experienced the same level of feather CORT deposition, regardless of the presence of damage. It is known that stress hormones like CORT in birds or cortisol in humans and other mammalian animals are important for the body's ability to respond to stress and injury. They are known to have complex effects on the skeleton, with small amounts needed for normal bone development but large amounts inhibiting bone growth (61). The finding of increased cumulative CORT deposition with age was similarly established in another recent chicken study (62). 
This in conjunction with the evidence in humans that prolonged treatment with glucocorticoids can produce osteoporosis (63), allowing us to speculate that our hens may produce osteoporosis characterized by a decrease in bone mass that may thus be related to KBD. In humans, it is reported (2) that bone adapts to stress with age, although its ability depends on both genetic factors and lifestyle, a phenomenon not yet proven in chickens. Nevertheless, knowing that the KB is typically reduced or absent in flightless birds $(64,65)$, likely as its main function is to provide adequate leverage for flight, and by assuming today's chickens are poor flyers (66) and very good egg producers, one can argue that the skeletal adaptation has changed with selection for high egg yield, increasing the frequency of KB breakage. Greater understanding of physiological and psychological stress-related relationships may help to reduce levels of damage and severity in modern chickens.

\section{CONCLUSIONS}

Psychosocial factors such as fear-induced pecking and locomotor reactions and sociality revealed an association with the development of an adverse bone condition in hens. Knowing that an individual's success with surviving and reproducing depends critically on its behavior, in the present work, we propose that hens with poor bone condition may experience psychological consequences from KBD but also that fear- and social-related psychological stress may be a potential predictor of bone damage.

\section{DATA AVAILABILITY STATEMENT}

The raw data supporting the conclusions of this article will be made available by the authors, without undue reservation.

\section{ETHICS STATEMENT}

All procedures with animals (experimental protocols and methods) were performed according to the legislation on animal

\section{REFERENCES}

1. Brenneman RE. Osteoporosis the silent disease. Prevention treatment of fragility fractures in a structured program. JLGH. (2016) 11:112-6. Available online at: http://www.jlgh.org/JLGH/media/Journal-LGH-Media-Library/ Past\%20Issues/Volume\%2011\%20-\%20Issue\%204/Osteoporosis-the-SilentDisease.pdf

2. U.S. Department of Health Human Services, Office of the Surgeon General. Bone Health Osteoporosis: A Report of the Surgeon General. Rockville, MD: Superintendent of Documents, U.S. Government Printing Office (2004).

3. Vellas BJ, Wayne SJ, Romero LJ, Baumgartner RN, Garry PJ. Fear of falling and restriction of mobility in elderly fallers. Age Ageing. (1997) 26:189-93. doi: 10.1093/ageing/26.3.189

4. Nees F, Witt SH, Flor H. Neurogenetic approaches to stress and fear in humans as pathophysiological mechanisms for posttraumatic stress disorder. Biol Psychiatry. (2018) 83:810-20. doi: 10.1016/j.biopsych.2017.12.015

5. Lay, Jr DC, Fulton RM, Hester PY, Karcher DM, Kjaer JB, et al. Hen welfare in different housing systems. Poult Sci. (2011) 90:278-94. doi: 10.3382/ps.2010-00962 experimentation in Slovenia and were approved by animalwelfare body at the Department of Animal Science, that is a member of Ethical Committee of the Administration of the Republic of Slovenia for Food Safety, Veterinary Sector and Plant Protection (UVHVVR) and were in accordance with the principals 3Rs.

\section{AUTHOR CONTRIBUTIONS}

NR contributed to the conceptualization, methodology, formal analysis, investigation, and writing the original draft. MZ contributed to the conceptualization, methodology, visualization, resources, writing, review and editing, and supervision. All authors contributed to the article and approved the submitted version.

\section{FUNDING}

This work was a part of the Slovene national project V4-1604 granted by the Slovenian Research Agency and Ministry of Agriculture, Forestry and Food (MKGP) Slovenia.

\section{ACKNOWLEDGMENTS}

The work considers the research topic covered within COST Action CA15224. We thank Ivan Dimitrov from the Agricultural Institute at Stara Zagora, Bulgaria, who visited our research center and was involved in the initial stage of study preparation. We are grateful to Dušan Terčič for technical assistance and logistical support, Nataša Debeljak for practical support, Špela Malovrh for initial statistical advice, and Nina Cebulj Kadunc from the Veterinary Faculty of the University of Ljubljana who provided laboratory facilities for corticosterone analyses. We wish to express our great gratitude to Sandra Edwards and Murray Bales for help with the English and for their comments on the manuscript. The research is part of the Master thesis of NR at the Department of Animal Science, Biotechnical Faculty of the University of Ljubljana, Slovenia.

6. Heerkens JL, Delezie E, Rodenburg TB, Kempen I, Zoons J, Ampe B, et al. Risk factors associated with keel bone and foot pad disorders in laying hens housed in aviary systems. Poult Sci. (2015) 95:482-8. doi: 10.3382/ps/p ev339

7. Stratmann A, Frohlich EKF, Harlander-Matausche A, Schrader L, Toscano MJ, Wurbel $\mathrm{H}$, et al. Soft perches in an aviary system reduce incidence of keel bone damage in laying hens. PLoS ONE. (2015) 10:e0122568. doi: 10.1371/journal.pone.0122568

8. Toscano MJ, Booth F, Wilkins LJ, Avery NC, Brown SB, Richards G, et al. The effects of long (c20/22) and short (c18) chain omega-3 fatty acids on keel bone fractures, bone biomechanics, behavior, egg production in free range laying hens. Poult Sci. (2015) 94:823-35. doi: 10.3382/ps/pev048

9. Riber AB, Casey-Trott TM, Herskin MS. The influence of keel bone damage on welfare of laying hens. Front Vet Sci. (2018) 5:6. doi: 10.3389/fvets.2018.00006

10. Toscano MJ, Dunn IC, Christensen JP, Petow S, Kittelsen K, Ulrich R. Explanations for keel bone fractures in laying hens: are there explanations in addition to elevated egg production? Poult Sci. (2020) 99:4183-94. doi: 10.1016/j.psj.2020.05.035 
11. Bain MM, Nys Y, Dunn IC. Increasing persistency in lay and stabilising egg quality in longer laying cycles. What are the challenges? Br Poult Sci. (2016) 57:330-8. doi: 10.1080/00071668.2016.1161727

12. Gebhardt-Henrich SG, Frohlich EK. Early onset of laying and bumblefoot favor keel bone fractures. Animals. (2015) 5:1192-206. doi: 10.3390/ani5040406

13. Nasr MA, Nicol CJ, Murrell JC. Do laying hens with keel bone fractures experience pain? PLoS ONE. (2012) 7:e42420. doi: 10.1371/journal.pone. 0042420

14. Darwin C. The variation of animals plants under domestication. In: Van Wyhe J, editor. The Complete Work of Charles Darwin Online. Darwin online, London: John Murray (1868). Available online at: http://darwin-online.org. $\mathrm{uk} /$ content/frameset?itemID=F878.1\&viewtype=text\&pageseq=1 (accessed April 10, 2019).

15. Warren DE. Physiological genetic studies of crooked keels in chickens. Kansas Agricultural Experiment Station Technical Bulletin. (1937). Available online at: https://www.ksre.k-state.edu/historicpublications/pubs/STB044. PDF (accessed December 15, 2018).

16. Winter AR, Funk EM. Poultry Science Practice. Philadelphia, PA: Lippincott, JB (1941).

17. Buckner GD, Insko WM Jr, Henry AH, Wachs EF. Rate of growth and calcification of the sternum of male and female new hampshire chickens. Poult Sci. (1948) 27:430-33. doi: 10.3382/ps.0270430

18. Casey-Trott T. Opportunities for exercise during pullet rearing: effects on bone health and keel bone damage in laying hens. (Dissertation), University of Guelph, Guelph, ON, Canada (2016).

19. Nasr MAF, Murrell J, Nicol CJ. The effect of keel fractures on egg production, feed and water consumption in individual laying hens. Br Poult Sci. (2013) 54:165-70. doi: 10.1080/00071668.2013.767437

20. Fraisse F, Cockrem JF. Corticosterone fear behaviour in white and brown caged laying hens. Br Poult Sci. (2006) 47:110-9. doi: 10.1080/00071660600610534

21. Ghareeb K, Niebuhr K, Awad WA, Waiblinger S, Troxler J. Stability of fear and sociality in two strains of laying hens. Br Poult Sci. (2008) 49:502-8. doi: 10.1080/00071660802290390

22. Edwards LE, Coleman GJ, Hemsworth PH. Close human presence reduces avoidance behaviour in commercial caged laying hens to an approaching human. Anim Prod Sci. (2013) 53:1276-82. doi: 10.1071/AN12342

23. Riber AB, Hinrichsen LK. Welfare consequences of omitting beak trimming in barn layers. Front Vet Sci. (2017) 4:222. doi: 10.3389/fvets.2017.00222

24. Armstrong EA, Rufener C, Toscano MJ, Eastham JE, Guy JH, Sandilands V, et al. Keel bone fractures induce a depressive-like state in laying hens. Sci Rep. (2020) 10:3007. doi: 10.1038/s41598-020-59940-1

25. Rauw WM, Johnson AK, Gomez-Raya L, Dekkers JCM. A hypothesis and review of the relationship between selection for improved production efficiency, coping behavior and domestication. Front Genet. (2017) 8:134. doi: 10.3389/fgene.2017.00134

26. de Haas EN, Kops MS, Bolhuis JE, Groothuis TG, Ellen ED, Rodenburg TB. The relation between fearfulness in young and stress-response in adult laying hens, on individual and group level. Physiol Behav. (2012) 107:433-9. doi: 10.1016/j.physbeh.2012.08.002

27. Bortolotti GR, Marchant TA, Blas J, German T. Corticosterone in feathers is a long-term, integrated measure of avian stress physiology. Funct Ecol. (2008) 22:494-500. doi: 10.1111/j.1365-2435.2008.0 1387.x

28. Šalehar A, Kompan D, Žan Lotrič M, Kovač M, Holcman A, Cepon M, et al. Razvoj Pasem Domačih Živali v Sloveniji: Prvotne, Izgubljene in Pretopljene Pasme. 2nd ed. Biotehniška fakulteta: Oddelek za zootehniko, Rodica, Slovenia (2014). 232p.

29. Jones RB. Fear adaptability in poultry: insights, implications imperatives. World Poult Sci J. (1996) 52:131-74. doi: 10.1079/WPS19960013

30. Harlander-Matauschek A, Rodenburg T, Sandilands V, Tobalske B, Toscano M. Causes of keel bone damage their solutions in laying hens. World Poult Sci J. (2015) 71:461-72. doi: 10.1017/S0043933915002135

31. Doherty WJ, DeRome ME, McCarthy MB, Gronowicz GA. The effect of corticosterone on osteoblast expression of beta 1 integrins. J Bone Joint Surg Am. (1995) 77:396-404. doi: 10.2106/00004623-199503000-00009
32. Genné-Bacon EA, Trinko JR, DiLeone RJ. Innate fear-induced weight regulation in the C57BL/6J mouse. Front Behav Neurosci. (2016) 10:132. doi: $10.3389 /$ fnbeh.2016.00132

33. Romero LM, Fairhurst GD. Measuring corticosterone in feathers: strengths, limitations, suggestions for the future. Comp Biochem Physiol A Mol Integr Physiol. (2016) 202:112-22. doi: 10.1016/j.cbpa.2016.05.002

34. Casey-Trott T, Heerkens JLT, Petrik M, Regmi P, Schrader L, Toscano MJ, et al. Methods for assessment of keel bone damage in poultry. Poult Sci. (2015) 94:2339-50. doi: 10.3382/ps/pev223

35. Gebhardt-Henrich SG, Rufener C, Stratmann A. Improving intra- interobserver repeatability and accuracy of keel bone assessment by training with radiographs. Poult Sci. (2019) 98:5234-40. doi: 10.3382/ps/pez410

36. de Haas EN, Lee C, Hernandez EC, Naguib M, Rodenburg BT. Individual differences in personality in laying hens are related to learning a colour cue association. Behav Processes. (2017) 134:37-42. doi: 10.1016/j.beproc.2016.11.001

37. Agnvall B, Jöngren M, Strandberg E, Jensen P. Heritability genetic correlations of fear-related behaviour in red junglefowl-possible implications for early domestication. PLoS ONE. (2012) 7:e35162. doi: 10.1371/journal.pone.0035162

38. Dawkins MS. Distance, social recognition in hens: implications for the use of photographs as social stimuli. Behaviour. (1996) 133:663-80. doi: 10.1163/156853996X00413

39. Jahn-Eimermacher A, Lasarzik I, Raber J. Statistical analysis of latency outcomes in behavioral experiments. Behav Brain Res. (2011) 221:271-5. doi: 10.1016/j.bbr.2011.03.007

40. Nakagawa S, Schielzeth $H$. Repeatability for gaussian and non-gaussian data: a practical guide for biologists. Biol Rev Camb Philos Soc. (2010) 85:935-56. doi: 10.1111/j.1469-185X.2010.00141.x

41. Weeks CA, Nicol CJ. Behavioural needs priorities preferences of laying hens. Worlds Poult Sci J. (2006) 62:296-307. doi: 10.1079/WPS2 00598

42. Geverink NA, Kappers A, Burgwal van de JA, Lambooij E, Blokhuis HJ, Wiegant VM. Effects of regular moving and handling on the behavioural and physiological consequences to preslaughter treatment and consequences for subsequent meat quality. J Anim Sci. (1998) 76:2080-5. doi: $10.2527 / 1998.7682080 \mathrm{x}$

43. Forkman B, Boissy A, Meunier-Salaün MC, Canali E, Jones RB. A critical review of fear tests used on cattle, pigs, sheep, poultry and horses. Physiol Behav. (2007) 92:340-74. doi: 10.1016/j.physbeh.2007.03.016

44. Buss AH. A theory of shyness. In: Jones WH, Cheek JM, Briggs SR, editors. Shyness. Perspectives on Research and Treatment. (Emotions, Personality, Psychotherapy). New York, NY: Springer Science+Business Media, Boston. (1986). p. 39-46. doi: 10.1007/978-1-4899-0525-3_4

45. Jones RB, Carcmichael N. Open-field behavior in domestic chicks tested individually or in pairs: differential effects of painted lines delineating subdivisions of the floor. Behav Res Methods Instrum Comput. (1997) 29:396400. doi: 10.3758/BF03200593

46. Mills DA, Jones BR, Faure MJ. Species specificity of social reinstatement in Japanese quail coturnix japonica genetically selected for high or low levels of social reinstatement behaviour. Behav Processes. (1995) 34:13-22. doi: 10.1016/0376-6357(94)00044-H

47. Zebunke M, Puppe B, Langbein J. Effects of cognitive enrichment on behavioural and physiological reactions of pigs. Physiol Behav. (2013) 118:709. doi: 10.1016/j.physbeh.2013.05.005

48. Rørvang MV, Christensen JW. Attenuation of fear through social transmission in groups of same and differently aged horses. Appl Anim Behav Sci. (2018) 209:41-6. doi: 10.1016/j.applanim.2018.10.003

49. Mills AD, Jones RB, Faure JM, Williams JB. Responses to isolation in Japanese quail genetically selected for high or low sociality. Physiol Behav. (1993) 53:183-9. doi: 10.1016/0031-9384(93)90029-F

50. Zupan M, Zanella AJ. Peripheral regulation of stress and fear responses in pigs from tail-biting pens. R Bras Zootec. (2017) 46:33-8. doi: 10.1590/s1806-92902017000100006

51. Jensen P, Toates FM. Who needs 'behavioural needs'? Motivational aspects of the needs of animals. Appl Anim Behav Sci. (1993) 37:161-81. doi: 10.1016/0168-1591(93)90108-2 
52. Koolhaas JM. Coping style immunity in animals: making sense of individual variation. Brain Behav Immun. (2008) 22:662-7. doi: 10.1016/j.bbi.2007.11.006

53. Koolhaas JM, Korte SM, De Boer SF, Van Der Vegt BJ, Van Reenen $\mathrm{CG}$, Hopster $\mathrm{H}$, et al. Coping styles in animals: current status in behavior and stress-physiology. Neurosci Biobehav Rev. (1999) 23:925-35. doi: 10.1016/S0149-7634(99)00026-3

54. de Haas EN, Lee C, Rodenburg TB. Learning judgment can be affected by predisposed fearfulness in laying hens. Front Vet Sci. (2017) 4:113. doi: $10.3389 /$ fvets.2017.00113

55. Ordiz A, Moen GK, Sæbø S, Stenset N, Swenson JE, Støen OG. Habituation sensitization, or consistent behavioral responses? Brown bear responses after repeated approaches by humans on foot. Biol Conserv. (2019) 232:228-37. doi: 10.1016/j.biocon.2019.01.016

56. Mitchell JE, Crow S. Medical complications of anorexia nervosa and bulimia nervosa. Curr Opin Psychiatry. (2006) 19:438-43. doi: 10.1097/01.yco.0000228768.79097.3e

57. Czirják TZ, Chereji A. Canine obesity - a major problem of pet dogs. Ecotox Zooteh Ind Alim. (2008) 7:361-6. Available online at: http://protmed.uoradea. ro/facultate/anale/ecotox_zooteh_ind_alim/2008/Czirjak\%20Zsolt.pdf

58. Stojcic MD, Bessei W. The effect of locomotor activity and weight load on bone problems in fast and slow growing chickens. Arch Geflügelkund. (2009) 73:242-9. Available online at: https://www.europeanpoultry-science.com/artikel.dll/m08-25mk_MTE2NzkxNg.PDF?UID= 904E30112A9F988C3E86AD9D4686BEB7EB28D0DA38C28F

59. Cao JJ. Effects of obesity on bone metabolism. J Orthop Surg Res. (2011) 6:30. doi: 10.1186/1749-799X-6-30

60. Toscano MJ, Booth F, Richards G, Brown SN, Karcher DM, Tarlton JF. Modeling collisions in laying hens as a tool to identify causative factors for keel bone fractures and means to reduce their occurrence and severity. PLoS ONE. (2018) 13:e0200025. doi: 10.1371/journal.pone.0200025

61. Canalis E, Delany AM. Mechanisms of glucocorticoid action in bone. Ann N Y Acad Sci. (2002) 966:73-81. doi: 10.1111/j.1749-6632.2002.tb04204.x

62. Nordquist RE, Zeinstra EC, Dougherty A, Riber AB. Effects of dark brooder rearing and age on hypothalamic vasotocin and feather corticosterone levels in laying hens. Front Vet Sci. (2020) 7:19. doi: 10.3389/fvets.2020.00019

63. Mazziotti G, Angeli A, Bilezikian JP, Canalis E, Giustina A. Glucocorticoidinduced osteoporosis: an update. Trends Endocrinol Metab. (2006) 17:144-9. doi: 10.1016/j.tem.2006.03.009

64. Gill FB. Ornithology. 2nd ed. Portland, OR: Freeman WH Company (1994).

65. Harshman J, Braun EL, Braun MJ, Edward L, Huddleston CJ, Bowie RCK, et al. Phylogenomic evidence for multiple losses of flight in ratite birds. Proc Natl Acad Sci USA. (2008) 105:13462-7. doi: 10.1073/pnas.0803242105

66. Ekesbo I. Farm Animal Behaviour: Characteristics for Assessment of Health Welfare. Wallingford; Cambridge: CABI (2011). doi: 10.1079/9781845937706.0000

Conflict of Interest: The authors declare that the research was conducted in the absence of any commercial or financial relationships that could be construed as a potential conflict of interest.

Copyright (c) 2020 Rokavec and Zupan Šmrov. This is an open-access article distributed under the terms of the Creative Commons Attribution License (CC BY). The use, distribution or reproduction in other forums is permitted, provided the original author(s) and the copyright owner(s) are credited and that the original publication in this journal is cited, in accordance with accepted academic practice. No use, distribution or reproduction is permitted which does not comply with these terms. 